\title{
Around a singular solution of a nonlocal nonlinear heat equation
}

\author{
Piotr Biler(1) and Dominika Pilarczyk
}

\begin{abstract}
We study the existence of global-in-time solutions for a nonlinear heat equation with nonlocal diffusion, power nonlinearity and suitably small data (either compared in the pointwise sense to the singular solution or in the norm of a critical Morrey space). Then, asymptotics of subcritical solutions is determined. These results are compared with conditions on the initial data leading to a finite time blowup.
\end{abstract}

Mathematics Subject Classification. 35K55, 35B05, 35B40, 60J60.

Keywords. Fractional Laplacian, Nonlinear heat equation, Singular solution, Global-in-time solutions, Singular potential, Asymptotic behavior, Stability.

\section{Introduction and main results}

Nonlinear evolution problems involving fractional Laplacian describing the anomalous diffusion (or the $\alpha$-stable Lévy diffusion) have been extensively studied in the mathematical and physical literature, see [43] for the Cauchy problem (1.1)-(1.2), and [9-12] for other examples of problems and for extensive list of references. One of these models is the following initial value problem for the reaction-diffusion equation with the anomalous diffusion

$$
\begin{aligned}
u_{t} & =-(-\Delta)^{\alpha / 2} u+|u|^{p-1} u, \quad \mathbb{R}^{d} \times(0, \infty), \\
u(x, 0) & =u_{0}(x),
\end{aligned}
$$

where the pseudodifferential operator $(-\Delta)^{\alpha / 2}$ with $0<\alpha \leqslant 2$ is defined by the Fourier transform: $\widehat{(-\Delta)^{\alpha} / 2}(\xi)=|\xi|^{\alpha} \widehat{u}(\xi)$. We will use the following wellknown integral definition of the fractional Laplacian with $\alpha \in(0,2)$, see e.g. $[13$, formula (1.4)],

The authors thank Grzegorz Karch and Philippe Souplet for interesting discussions. We are indebted to the referee for pertinent comments. The first author has been partially supported by the NCN Grant 2016/23/B/ST1/00434. 


$$
-(-\Delta)^{\alpha / 2} f(x)=\mathcal{A} \lim _{\delta \searrow 0} \int_{\{|y|>\delta\}} \frac{f(x-y)-f(x)}{|y|^{d+\alpha}} \mathrm{d} y,
$$

where the constant is, by e.g. [13, formula (1.5)],

$$
\mathcal{A}=\mathcal{A}(d, \alpha)=\frac{2^{\alpha} \Gamma\left(\frac{d+\alpha}{2}\right)}{\pi^{\frac{d}{2}}\left|\Gamma\left(-\frac{\alpha}{2}\right)\right|} .
$$

Moreover, we assume that $p>1$ and $u_{0}(x) \geqslant 0$.

Nonnegative solutions of the linear fractional heat equation $v_{t}=-$ $(-\Delta)^{\alpha / 2} v$ have been studied extensively. A complete counterpart of the Widder theory of nonnegative solutions of the classical linear heat equation is presented in $[4,18]$. Namely, existence, uniqueness and the characterization of initial traces as measures satisfying an integrability condition are given.

This paper is a straightforward generalization of extensively studied classical nonlinear heat equation, see [40], to the case of nonlocal but linear diffusion operators defined by fractional powers of Laplacian. Study of solutions around a critical singular solution which is not smoothed out by the diffusion is, in a sense, parallel to analyses for nonlinear heat equation in [40] and, in particular, in $[37,38]$. This also reveals some threshold phenomena as is in the former case.

\subsection{Statement of results}

The form of singular steady state $u_{\infty}$ of equation (1.1) is given in Proposition 2.1. Existence of global-in-time solutions for initial data in a suitable critical Morrey space is shown in Proposition 2.3. Local-in-time existence of solutions for initial data having local singularities weaker than $u_{\infty}$ is proved in Proposition 2.5.

Global-in-time existence of solutions for subcritical initial data $u_{0} \leqslant u_{\infty}$ is derived in Theorem 2.6, and this construction is based on a novel comparison principle for special solutions in Proposition 2.7. These solutions have diffusion dominated asymptotics.

Analysis of the linearization operator (nonlocal diffusion + Hardy-type potential) around the singular state in Sect. 3 leads to its nonlinear $L^{2}$ asymptotic stability. Fine asymptotics of solutions in vicinity of $u_{\infty}$ is given.

Finally, we interpret results on finite time blowup of solutions with "large" initial data (in [43] and recently [7]) as a kind of partial dichotomy, see Corollary 4.1 .

Concerning the question of nonexistence of global-in-time solutions to equation (1.1), the first results have been proved in [43] with the argument based on the seminal idea of [27]. Extensions of such blowup results for equations with linear but nonlocal diffusion operators more general than fractional Laplacians and localized source terms are in a forthcoming paper [7] which improves some results in [1] and clarifies sufficient conditions for blowup. Interpretations of sufficient blowup conditions in terms of a quantity like the Morrey space $M^{d(p-1) / \alpha}\left(\mathbb{R}^{d}\right)$ norm in [7] lead to Corollary 4.1. Remark that the discrepancy bounds between sufficient bounds for global-in-time existence 
and sufficient bounds for blowup are quite well controlled for large dimensions $d$, see [7, Theorem 5].

\subsection{Notation}

In the sequel, $\|\cdot\|_{p}$ denotes the usual $L^{p}\left(\mathbb{R}^{d}\right)$ norm, and $C$ 's are generic constants independent of $t, u, \ldots$ which may, however, vary from line to line. The (homogeneous) Morrey spaces over $\mathbb{R}^{d}$ modeled on $L^{q}\left(\mathbb{R}^{d}\right), q \geqslant 1$, are defined by their norms

$$
\|u\|_{M_{q}^{s}} \equiv\left(\sup _{R>0, x \in \mathbb{R}^{d}} R^{d(q / s-1)} \int_{\{|y-x|<R\}}|u(y)|^{q} \mathrm{~d} y\right)^{1 / q}<\infty .
$$

Caution: the notation for Morrey spaces used elsewhere might be different, e.g. $M_{q}^{s}$ is denoted by $M^{q, \lambda}$ with $\lambda=d q / s$ in [41].

The most frequent situation is when $q=1$ and we consider $M_{1}^{s} \equiv M^{s}$. The spaces $M^{d(p-1) / \alpha}\left(\mathbb{R}^{d}\right)$ and more general $M_{q}^{d(p-1) / \alpha}\left(\mathbb{R}^{d}\right), q>1$, are critical in the study of equation (1.1), see [41]. We refer the readers to $[5,15,33]$ for analogous examples in chemotaxis theory.

Integrals with no integration limits are meant to be calculated over the whole space $\mathbb{R}^{d}$. The asymptotic relation $f \approx g$ means that $\lim _{s \rightarrow \infty} \frac{f(s)}{g(s)}=1$ and $f \asymp g$ is used whenever $\lim _{s \rightarrow \infty} \frac{f(s)}{g(s)} \in(0, \infty)$.

\section{Existence of global-in-time solutions below the singular solution}

\subsection{Existence of the singular solution}

We have the following

Proposition 2.1. For $p>1+\frac{\alpha}{d-\alpha}$, there is a unique radial homogeneous nonnegative solution of the equation

$$
(-\Delta)^{\alpha / 2} u_{\infty}=u_{\infty}^{p}, \quad \text { with } u_{\infty}(x)=s(\alpha, d, p)|x|^{-\frac{\alpha}{p-1}}
$$

with the constant

$$
s(\alpha, d, p)=\left(\frac{2^{\alpha}}{\Gamma\left(\frac{\alpha}{2(p-1)}\right)} \frac{\Gamma\left(\frac{d}{2}-\frac{\alpha}{2(p-1)}\right) \Gamma\left(\frac{p \alpha}{2(p-1)}\right)}{\Gamma\left(\frac{d}{2}-\frac{p \alpha}{2(p-1)}\right)}\right)^{\frac{1}{p-1}} .
$$

Proof. By formula (1.3) (see also $[13,(3.6)]$ ) for the fractional Laplacian, we have for $d>\frac{p}{p-1} \alpha$, i.e. $p>1+\frac{\alpha}{d-\alpha}$

$$
\frac{\alpha}{p-1}+2 \frac{\alpha}{2}=p \frac{\alpha}{p-1} \text {. }
$$

Thus, a multiple of the function $|x|^{-\alpha /(p-1)}$ is a solution of equation (2.1). The value of $s(\alpha, d, p)$ is determined from the convolution identities $[13,(3.3)]$, see also formula $(2.20)$. The solution $u_{\infty}$ satisfies $\left\|u_{\infty}\right\|_{M^{d(p-1) / \alpha}}=\frac{\sigma_{d}}{d-\frac{\alpha}{p-1}}$ $s(\alpha, d, p)$. 
Remark 2.2. If $\alpha=2$ it is known that the exponent $p_{\mathrm{sg}}=1+\frac{2}{d-2}$ is critical for the existence of a positive, radial, distributional solution $u_{\infty}(x)=$ $\left(\frac{2}{p-1}\left(d-2-\frac{2}{p-1}\right)\right)^{\frac{1}{p-1}}|x|^{-\frac{2}{p-1}}$ of the equation $\Delta u+u^{p}=0$ in $\mathbb{R}^{d}$ (see for example [40] and references therein). In the case $\alpha \in(0,2)$ the analogue of this exponent is $1+\frac{\alpha}{d-\alpha}$, see Remark 3.2. For some smooth solutions of equation (2.1), see [16].

\subsection{Small global-in-time solutions}

Our purpose is to prove two global in time existence results, the first one under smallness condition of the norm of critical homogeneous Morrey space $M_{q}^{d(p-1) / \alpha}\left(\mathbb{R}^{d}\right)$ with some $q>1$, and the second under the assumption that the initial data $u_{0}$ is below the singular solution $u_{\infty}$ in the pointwise sense.

We will use the technique of Picard iterations of constructing mild solutions, i.e. those satisfying the Duhamel formula

$$
u(t)=\mathrm{e}^{-t(-\Delta)^{\alpha / 2}} u_{0}+\int_{0}^{t} \mathrm{e}^{-(t-\tau)(-\Delta)^{\alpha / 2}}\left(|u|^{p-1} u\right)(\tau) \mathrm{d} \tau .
$$

A systematic approach to the existence of mild solutions via iterations of nonlinear mappings, as those on the right-hand side of formula (2.3), for various evolution equations (motivated mainly by hydrodynamics and chemotaxis theory) is presented in full detail in, e.g., [32,33].

Proposition 2.3. If $p>1+\frac{\alpha}{d}$, i.e. $\frac{d(p-1)}{\alpha}>1$, and the initial condition $u_{0}$ is sufficiently small in the sense of the norm of the homogeneous Morrey space $M_{q}^{d(p-1) / \alpha}\left(\mathbb{R}^{d}\right)$ with some $q>1$, then solution of problem $(1.1)-(1.2)$ is global in time.

Proof. For $\alpha=2$, if the norm $\left|u_{0}\right|_{M_{q}^{d(p-1) / \alpha}}$ (for a number $q>1$ ) is small enough, then a solution of problem (1.1)-(1.2) is global in time, see [41, Proposition 6.1] (as well as a counterpart for the chemotaxis system cf. [5, Th. 1]). Similarly as the former result, it can be proved directly for $\alpha \in(0,2)$ (while the proof in [41] for $\alpha=2$ was by contradiction) using the Picard iterations of the mapping

$$
\mathcal{N}(u)(t)=\mathrm{e}^{-t(-\Delta)^{\alpha / 2}} u_{0}+\int_{0}^{t} \mathrm{e}^{-(t-\tau)(-\Delta)^{\alpha / 2}}\left(|u|^{p-1} u\right)(\tau) \mathrm{d} \tau,
$$

$u_{n+1}=\mathcal{N}\left(u_{n}\right), n=1,2, \ldots$, with $u_{0} \in M_{q}^{d(p-1) / \alpha}\left(\mathbb{R}^{d}\right)(q>1, p>1+2 q / d)$ small enough. For useful estimates of the heat semigroup in Morrey spaces we refer the reader to [29] and [44, Th. 3.8]. They extend immediately to the estimate for $1<p_{1}<p_{2}<\infty$

$$
\left\|\mathrm{e}^{-t(-\Delta)^{\alpha / 2}} f\right\|_{M_{q_{2}}^{p_{2}}} \leqslant C t^{-d\left(1 / p_{1}-1 / p_{2}\right) / \alpha} \|\left. f\right|_{M_{q_{1}}^{p_{1}}}
$$

which holds with $q_{2} / q_{1}=p_{2} / p_{1}$ if $p_{1}<d$. Thus, we have

$$
\mathrm{e}^{-\cdot(-\Delta)^{\alpha / 2}}: M_{q}^{s}\left(\mathbb{R}^{d}\right) \rightarrow \mathcal{Y} \equiv\left\{u: \sup _{t>0} t^{-\beta}|u(t)|_{M_{r}^{r s / q}}<\infty\right\}
$$


with $\beta=\frac{d}{\alpha}\left(1-\frac{q}{r}\right)=\left(1-\frac{q}{r}\right) \frac{1}{p-1}$. When either $p_{1}=1$ or $p_{2}=\infty$, the norms are $\|\cdot\|_{1}$ and $\|\cdot\|_{\infty}$, resp. The crucial estimate for the convergence of the Picard iterations is

$$
\left\|\int_{0}^{t} \mathrm{e}^{-(t-\tau)(-\Delta)^{\alpha / 2}}\left(|u|^{p-1} u\right)(\tau) \mathrm{d} \tau\right\|_{M_{r}^{r d(p-1) /(\alpha q)}} \leqslant t^{-\beta}\|u\|^{p},
$$

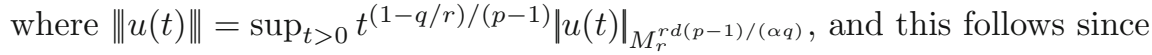

$$
\int_{0}^{t}(t-\tau)^{-\frac{d}{\alpha}(q p / r s-q / r s)} \tau^{-p \beta} \mathrm{d} \tau=C t^{-\beta}
$$

for some constant $C>0$ since $-\frac{d}{\alpha s} \frac{q}{r}(p-1)-p \beta+1=-\frac{q}{r}+p \beta+1=-\beta$.

They are convergent in the norm $\|\cdot\|$ for $\max \{p, q\}<r<p q$, since $\left\|\mathrm{e}^{-t(-\Delta)^{\alpha / 2}} u_{0}\right\|<\infty$. Clearly, assumption (2.9) in Th. 2.6 implies $\left|u_{0}\right|_{M^{d(p-1) / \alpha}}$ $<\frac{\sigma_{d}}{d-\frac{\alpha}{p-1}} \varepsilon s(\alpha, d, p)$, and the conclusion (2.14) reads $\|\left. u(t)\right|_{M^{d(p-1) / \alpha}}<\frac{\sigma_{d}}{d-\frac{\alpha}{p-1}} \varepsilon$ $s(\alpha, d, p)$.

Then, usual bootstrapping arguments like in [40, proof of Th. 15.2, p. 81] apply and $u(t) \in L^{\infty}$ follows for each $t>0$. The authors are indebted to Philippe Souplet for this argument showing regularity. Note that this existence result remains valid for nonlinearities with behavior $|F(u)| \asymp|u|^{p}$ as $u \rightarrow 0$.

Remark 2.4. Let us recall that for $\alpha=2$ the number $p_{F}=1+\frac{2}{d}$, called the Fujita exponent, borders the case of a finite-time blowup for all positive solutions (for $p \leqslant p_{F}$ ) and the case of existence of some global in time bounded positive solutions (if $p>p_{F}$ ) (see for example [40] and references therein). The exponent $1+\frac{\alpha}{d}$ is a counterpart of the Fujita exponent in the case $\alpha \in(0,2)$, see [43].

In the next proposition $u_{0}$ is supposed to have asymptotics for large $|x|$ like $u_{\infty}(x) \asymp \frac{1}{|x|^{2}}$ but local singularities strictly weaker since $u_{0} \in M^{\tilde{s}}\left(\mathbb{R}^{d}\right)$ with some $\tilde{s}>s$ is assumed.

Proposition 2.5. If $p>1+\frac{\alpha}{d}, s=\frac{d(p-1)}{\alpha}<\tilde{s}$ and $u_{0} \in M^{s}\left(\mathbb{R}^{d}\right) \cap M^{\tilde{s}}\left(\mathbb{R}^{d}\right)$, then there exists $T>0$ and a local in time solution

$$
\begin{aligned}
u \in \mathcal{X}_{T} & \equiv \mathcal{C}\left([0, T] ; M^{s}\left(\mathbb{R}^{d}\right) \cap M^{\tilde{s}}\left(\mathbb{R}^{d}\right)\right) \\
& \cap\left\{u:(0, T) \rightarrow L^{\infty}\left(\mathbb{R}^{d}\right): \sup _{0<t<T} t^{\frac{d}{\alpha \tilde{s}}}\|u(t)\|_{\infty}<\infty\right\}
\end{aligned}
$$

of problem (1.1)-(1.2).

Proof. We follow the approach and notations in the proof of Proposition 2.3. We will estimate the nonlinear operator $\mathcal{N}(u)$ in the norms of the spaces $M^{s}\left(\mathbb{R}^{d}\right), M^{\tilde{s}}\left(\mathbb{R}^{d}\right)$ and $\mathcal{Y}_{T} \equiv\left\{u:(0, T) \rightarrow L^{\infty}\left(\mathbb{R}^{d}\right): \sup _{0<t<T} t^{\frac{d}{\alpha \tilde{s}}}\|u(t)\|_{\infty}\right.$ $<\infty\}$. 
The first estimate is in the $M^{s}\left(\mathbb{R}^{d}\right)$ norm

$$
\begin{aligned}
|\mathcal{N}(u)(t)|_{M^{s}} & \leqslant C \int_{0}^{t}\|u(\tau)\|_{M^{s}}\|u(\tau)\|_{\infty}^{p-1} \mathrm{~d} \tau \\
& \leqslant C \int_{0}^{t}\|u(\tau)\|_{M^{s}} \tau^{-\frac{d}{\alpha \tilde{s}}(p-1)}\|u\|_{\mathcal{Y}_{T}}^{p-1} \mathrm{~d} \tau \\
& \leqslant C T^{1-\frac{d}{\alpha \tilde{s}}(p-1)} \sup _{t \in(0, T)}\|u(t)\|_{M^{s}}\|u\|_{\mathcal{Y}_{T}}^{p-1}
\end{aligned}
$$

since $\frac{d}{\alpha \tilde{s}}(p-1)<\frac{d}{\alpha s}(p-1)=1$.

The second estimate is in the $M^{\tilde{s}}\left(\mathbb{R}^{d}\right)$ norm

$$
\begin{aligned}
\|\mathcal{N}(u)(t)\|_{M^{\tilde{s}}} & \leqslant C \int_{0}^{t}(t-\tau)^{-\frac{d}{\alpha}\left(\frac{1}{s}-\frac{1}{\tilde{s}}\right)}\|u(\tau)\|_{M^{s}}\|u(\tau)\|_{\infty}^{p-1} \mathrm{~d} \tau \\
& \leqslant C \int_{0}^{t}(t-\tau)^{-\frac{d}{\alpha}\left(\frac{1}{s}-\frac{1}{\tilde{s}}\right)}\|u(\tau)\|_{M^{s}} \tau^{-\frac{d}{\alpha \tilde{s}}(p-1)}\|u\|_{\mathcal{Y}_{T}}^{p-1} \mathrm{~d} \tau \\
& \leqslant C T^{1-\frac{d}{\alpha}\left(\frac{1}{s}-\frac{1}{\tilde{s}}\right)-\frac{d}{\alpha \tilde{s}}(p-1)} \sup _{t \in(0, T)} \mid u(t)\left\|_{M^{s}}\right\| u \|_{\mathcal{Y}_{T}}^{p-1}
\end{aligned}
$$

since $1-\frac{d}{\alpha}\left(\frac{1}{s}-\frac{1}{\tilde{s}}\right)-\frac{d}{\alpha \tilde{s}}(p-1)=1-\frac{1}{p-1}-\frac{d}{\alpha \tilde{s}}(p-2)>0$.

The third estimate is

$$
\begin{aligned}
t^{\frac{d}{\alpha \tilde{s}}}\|\mathcal{N}(u)(t)\|_{\infty} & \leqslant C t^{\frac{d}{\alpha \tilde{s}}} \int_{0}^{t}(t-\tau)^{-\frac{d}{\alpha s}} \tau^{-\frac{d}{\alpha \tilde{s}}(p-1)} \mathrm{d} \tau\|u\|_{\mathcal{Y}_{T}}^{p} \\
& \leqslant C T^{\nu}\|u\|_{\mathcal{Y}_{T}}^{p}
\end{aligned}
$$

with $\nu=\frac{d}{\alpha \tilde{s}}-\frac{d}{\alpha s}-\frac{d}{\alpha \tilde{s}}(p-1)+1>0$. These bounds (2.6)-(2.8) lead in a standard way to the convergence of the Picard iterations for initial data in $M^{s}\left(\mathbb{R}^{d}\right) \cap M^{\tilde{s}}\left(\mathbb{R}^{d}\right)$ of arbitrary size and for sufficiently small $t>0$.

\subsection{Large global-in-time solutions}

The main result in this section is

Theorem 2.6. If $\alpha \in(0,2), p>1+\frac{\alpha}{d-\alpha}\left(>1+\frac{\alpha}{d}\right), u=u(x, t)$ is a solution of problem (1.1)-(1.2) with the initial data satisfying $u_{0} \in M^{\tilde{s}}\left(\mathbb{R}^{d}\right)$ for some $\tilde{s}>\frac{d(p-1)}{\alpha}$ and

$$
0 \leqslant u_{0}(x) \leqslant u_{\infty}(x)
$$

as well as

$$
\lim _{|x| \rightarrow \infty}|x|^{\frac{\alpha}{p-1}} u(x, t)=0 \quad \text { uniformly in } t \in(0, T) .
$$

Then $u$ can be continued to a global-in-time solution which still satisfies the bound

$$
0 \leqslant u(x, t) \leqslant u_{\infty}(x) .
$$

Condition (2.9) means that $u_{0} \in M^{s}\left(\mathbb{R}^{d}\right)$ with $s=\frac{d(p-1)}{\alpha}$. Thus, $\tilde{s}>s$ can be chosen as close to $\frac{d(p-1)}{\alpha}$ as we wish. Therefore, Proposition 2.5 on local-in-time solutions applies to those data. 
The result in Theorem 2.6 is based on the following restricted comparison principle, see [13, Th. 4.1, Th. 5.1] for analogous albeit more complicated constructions for radially symmetric solutions of chemotaxis systems.

Proposition 2.7. For each $\delta \in(0,1)$ and each $K>0$ there exist $\gamma_{0} \in\left(0, \frac{\alpha}{p-1}\right)$ (independent of $K$ and sufficiently close to $\frac{\alpha}{p-1}$ ) such that every solution $u \in$ $\mathcal{C}^{1}\left(\mathbb{R}^{d} \times(0, T]\right)$ with the property

$$
\lim _{|x| \rightarrow \infty}|x|^{\frac{\alpha}{p-1}} u(x, t)=0 \quad \text { uniformly in } \quad t \in(0, T),
$$

and the initial data satisfying

$$
0 \leqslant u_{0}(x)<\min \left\{\frac{K}{|x|^{\gamma_{0}}}, \frac{\delta s(\alpha, d, p)}{|x|^{\frac{\alpha}{p-1}}}\right\} \equiv b(x),
$$

satisfies the estimate

$$
0 \leqslant u(x, t)<b(x) \text { for all } x \in \mathbb{R}^{d} \text { and } 0<t \leqslant T .
$$

Once the comparison principle in Proposition 2.7 is proved, the localin-time solution constructed in Proposition 2.5 can be continued onto some interval $[T, T+h]$, and further, step-by-step with the same $h>0$, onto the whole half-line $[0, \infty)$ to a global-in-time solution satisfying the bound $u(x, t)<$ $b(x)$. Indeed, if inequality (2.13) holds, then $u \in M^{\tilde{s}}\left(\mathbb{R}^{d}\right)$ for some $\tilde{s}>\frac{d(p-1)}{\alpha}$, and clearly $u_{0} \in M^{\frac{d(p-1)}{\alpha}}\left(\mathbb{R}^{d}\right)$ by $0 \leqslant u_{0}(x) \leqslant u_{\infty}(x)$. By Proposition 2.5 , the solution $u$ is locally in time in $L^{\infty}\left(\mathbb{R}^{d}\right)$, hence smooth by standard arguments.

Proof of Theorem 2.6. Approximating $u_{0}$ in Theorem 2.6 by initial data $u_{0 k}(x)=\min \left\{u_{0}(x),(1-1 / k) u_{\infty}(x)\right\}$ satisfying (2.13) with $\delta=1-1 / k, k=$ $2,3,4, \ldots$, we obtain a global-in-time solution via a monotonicity argument. This procedure is an adaptation of the monotone approximation argument for the classical nonlinear heat equation in [28]. The first step is to show the property that $u_{k}$ increase with $k$. This can be done in standard way writing the equation for the difference $w$ of two approximating solutions $w=u_{l}-u_{k}$, $l>k$, as

$$
w_{t}=-(-\Delta)^{\alpha / 2} w+\frac{u_{l}^{p}-u_{k}^{p}}{u_{l}-u_{k}} w .
$$

This is a linear equation of the type $w_{t}=-(-\Delta)^{\alpha / 2} w+V(x) w$ with $0 \leq$ $V(x) \leq C|x|^{-\alpha}$ considered in the next section. The associated semigroup conserves positivity of the initial data $w_{0}$. Then, the pointwise monotone limit of $u_{k}$ 's exists and satisfies equation (1.1) in the weak sense (using the Lebesgue dominated convergence theorem).

We sketch the proof of the comparison principle.

Proof of Proposition 2.7. Let $u$ be a solution of problem (1.1)-(1.2) for an initial data satisfying (2.13). The proof of inequality (2.14) is by contradiction. Suppose a contrario that there exists $t_{0} \in(0, T]$ which is the first moment when $u(x, t)$ hits the barrier $b(x)$ defined in $(2.13)$. By a priori $\mathcal{C}^{1}$ regularity of $u(x, t)$ 
and by property (2.12) the value of $t_{0}$ is well defined. Moreover, there exists $x_{0} \in \mathbb{R}^{d}$ satisfying $u\left(x_{0}, t_{0}\right)=b\left(x_{0}\right)$. Define the number

$$
R_{\#}=\left(\frac{\delta s(\alpha, d, p)}{K}\right)^{1 /\left(\gamma_{0}-\frac{\alpha}{p-1}\right)}
$$

where two parts of the graph of the barrier $b$ meet. Let us consider the auxiliary function

$$
\tilde{u}\left(x, t_{0}\right)=|x|^{\gamma} u\left(x, t_{0}\right) \text { with } \gamma=\left\{\begin{array}{lll}
\frac{\alpha}{p-1} & \text { if } \quad\left|x_{0}\right| \geq R_{\#}, \\
\frac{d}{\tilde{s}}<\frac{\alpha}{p-1} & \text { if } \quad 0 \leqslant R_{\#} \leq\left|x_{0}\right| .
\end{array}\right.
$$

With this choice $\tilde{u}$ hits a constant part of the graph of the modified barrier $|x|^{\gamma} b(x)$. Thus, $\tilde{u}\left(x, t_{0}\right)$ attains its local maximum at $x_{0}$. Indeed, the existence of an $x_{1} \neq x_{0}$ such that $\tilde{u}\left(x_{1}, t_{0}\right)>\tilde{u}\left(x_{0}, t_{0}\right)$ would contradict the choice of $t_{0}$ as the first hitting point of the barrier. Clearly, $\nabla \tilde{u}\left(x_{0}, t_{0}\right)=0$ and $\frac{\partial}{\partial t} \tilde{u}\left(x_{0}, t_{0}\right) \geqslant 0$ hold. We will show that nevertheless $\left.\frac{\partial}{\partial t} \tilde{u}\left(x_{0}, t\right)\right|_{t=t_{0}}<0$, a contradiction.

Now, let us compute according to formula (1.3) for the fractional Laplacian

$$
\begin{aligned}
\frac{\partial}{\partial t} \tilde{u} & \left.\left(x_{0}, t\right)\right|_{t=t_{0}} \\
= & \left|x_{0}\right|^{\gamma}\left(-(-\Delta)^{\alpha / 2} u+u^{p}\right) \\
= & \left|x_{0}\right|^{\gamma}\left(-\left.(-\Delta)^{\alpha / 2}\left(|x|^{-\gamma} \tilde{u}\left(x, t_{0}\right)\right)\right|_{x=x_{0}}+\left(\left|x_{0}\right|^{-\gamma} \tilde{u}\left(x_{0}, t_{0}\right)\right)^{p}\right) \\
= & \left|x_{0}\right|^{\gamma} P . V \cdot \mathcal{A} \int \frac{\left|x_{0}\right|^{\gamma} \tilde{u}\left(x_{0}-y, t_{0}\right)-\left|x_{0}-y\right|^{\gamma} \tilde{u}\left(x_{0}, t\right)}{\left|x_{0}\right|^{\gamma}\left|x_{0}-y\right|^{\gamma}|y|^{d+\alpha}} \mathrm{d} y \\
& +\left|x_{0}\right|^{-(p-1) \gamma} \tilde{u}\left(x_{0}, t_{0}\right)^{p} \\
= & \left|x_{0}\right|^{\gamma} \tilde{u}\left(x_{0}, t_{0}\right) P \cdot V \cdot \mathcal{A} \int \frac{1}{|y|^{d+\alpha}}\left(\frac{1}{\left|x_{0}-y\right|^{\gamma}}-\frac{1}{\left|x_{0}\right|^{\gamma}}\right) \mathrm{d} y \\
& +\left|x_{0}\right|^{\gamma} P . V \cdot \mathcal{A} \int \frac{\tilde{u}\left(x_{0}-y, t_{0}\right)-\tilde{u}\left(x_{0}, t_{0}\right)}{\left|x_{0}-y\right|^{\gamma}|y|^{d+\alpha}} \mathrm{d} y+\left|x_{0}\right|^{-(p-1) \gamma} \tilde{u}\left(x_{0}, t_{0}\right)^{p} \\
\leqslant & \tilde{u}\left(x_{0}, t_{0}\right)\left(\left|x_{0}\right|^{\gamma}(-\Delta)^{\alpha / 2}\left(|x|^{-\gamma}\right)_{\mid x=x_{0}}+\left|x_{0}\right|^{-(p-1) \gamma} \tilde{u}\left(x_{0}, t_{0}\right)^{p-1}\right)
\end{aligned}
$$

The passage from (2.17) to (2.18) is obvious, see an analogous reasoning in [13, (4.19)] which involves definition (1.3) above. Formula [13, (3.6)] obtained for $\alpha+\gamma<d$ from (1.3) and convolution identities for powers $|x|^{-\gamma}$, etc., $[13,(3.3)]$

$$
(-\Delta)^{\alpha / 2}\left(|x|^{-\gamma}\right)=2^{\alpha} \frac{\Gamma\left(\frac{d-\gamma}{2}\right) \Gamma\left(\frac{\alpha+\gamma}{2}\right)}{\Gamma\left(\frac{d-\alpha-\gamma}{2}\right) \Gamma\left(\frac{\gamma}{2}\right)}|x|^{-\alpha-\gamma}
$$

gives for $\gamma=\frac{\alpha}{p-1}$ (so that $\alpha+\gamma<d$ by assumption $\left.p>1+\frac{\alpha}{d-\alpha}\right)$

$$
(-\Delta)^{\alpha / 2}\left(|x|^{-\frac{\alpha}{p-1}}\right)=2^{\alpha} \frac{\Gamma\left(\frac{d-\frac{\alpha}{p-1}}{2}\right) \Gamma\left(\frac{p \alpha}{2(p-1)}\right)}{\Gamma\left(\frac{d-\frac{p \alpha}{p-1}}{2}\right) \Gamma\left(\frac{\alpha}{2(p-1)}\right)}|x|^{-\frac{p \alpha}{p-1}} .
$$


We use this passing from (2.18) to (2.19). In the first case when $\gamma=\frac{\alpha}{p-1}$, we recall formula $(2.2)$ for the constant $s(\alpha, d, p)$ which leads to

$\tilde{u}\left(x_{0}, t_{0}\right)^{p-1}=\delta^{p-1} s(\alpha, d, p)^{p-1}=\delta^{p-1} \frac{2^{\alpha}}{\Gamma\left(\frac{\alpha}{2(p-1)}\right)} \frac{\Gamma\left(\frac{d}{2}-\frac{\alpha}{2(p-1)}\right) \Gamma\left(\frac{p \alpha}{2(p-1)}\right)}{\Gamma\left(\frac{d}{2}-\frac{p \alpha}{2(p-1)}\right)}$.

The passage from (2.18) to (2.19) in the middle term follows since $\tilde{u}\left(., t_{0}\right)$ assumes its maximal value at $x_{0}$, and (2.19) equals

$$
\tilde{u}\left(x_{0}, t_{0}\right)\left|x_{0}\right|^{-\alpha} s(\alpha, d, p)^{p-1}\left(-1+\delta^{p-1}\right)<0 .
$$

The obtained inequality $\left.\frac{\partial}{\partial t} \tilde{u}\left(x_{0}, t\right)\right|_{t=t_{0}}<0$ contradicts the assumption that $\tilde{u}$ hits for the first time the constant level $\delta s(\alpha, d, p)$ at $t=t_{0}$.

In the second case when $\gamma<\frac{\alpha}{p-1}$ but close to $\frac{\alpha}{p-1}$

$$
\tilde{u}\left(x_{0}, t_{0}\right)^{p-1}=\left(K\left|x_{0}\right|^{-\gamma}\right)^{p-1} \leqslant\left(\delta s(\alpha, d, p)\left|x_{0}\right|^{-\frac{\alpha}{p-1}}\right)^{p-1}
$$

by the definition of the barrier (2.13). Since the coefficient in formula (2.20) is continuous when $\gamma \nearrow \frac{\alpha}{p-1}$, so $(p-1) \gamma \nearrow \alpha$, the right-hand side of inequality (2.19) is strictly negative for $\gamma$ close enough to $\frac{\alpha}{p-1}$. This happens for $\gamma \in\left(\gamma_{0}, \frac{\alpha}{p-1}\right)$ with $\gamma_{0}=\gamma_{0}\left(\delta, \frac{\alpha}{p-1}\right)$ independent of other parameters.

Remark 2.8. This kind of result in Theorem 2.6 is known for the classical nonlinear heat equation (cf. [31, Th. A]) but the proof in [6] seems be somewhat novel. Similar pointwise arguments are powerful tools and, as such, they have been used in different contexts as e.g. fluid dynamics and chemotaxis theory: $[19,21,22,30]$, and free boundary problems. If $u_{0}$ is radially symmetric and $u_{0}(x)<\delta u_{\infty}(x)$ for some $\delta<1$, then the solution of (1.1)-(1.2) exists globally in time, see [36, Theorem 1.1] and also [41, Remark 3.1(iv)]. Related results are in [35, Lemma 2.2], and stability of the singular solution is studied in [39]. Results for not necessarily radial solutions starting either below or slightly above the singular solution $u_{\infty}$ are in [28, Th. 10.4] (reported in [40, Th. 20.5]), and in [42, Th. 1.1]. Note that solutions of the Cauchy problem for equation (1.1) for $\alpha=2$ in the latter case are nonunique.

Here, these considerations have been extended to the case of diffusion operators in equation (1.1) defined in a nonlocal way, similarly as it had been done in [13, Theorem 2.5] for radial solutions of the chemotaxis system with $\alpha \in(0,2)$.

Remark 2.9. (decay estimates for global in time solutions) The construction of solutions in Theorem 2.6 as perturbations of the solution $\mathrm{e}^{-t(-\Delta)^{\alpha / 2}} u_{0}$ to the linear equation $u_{t}+(-\Delta)^{\alpha / 2} u=0$ leads to the asymptotic estimate

$$
\|u(t)\|_{\infty}=\mathcal{O}\left(t^{-1 /(p-1)}\right) \text { when } t \rightarrow \infty,
$$

exactly the same as for solutions to that equation with general positive initial data in $M^{d(p-1) / \alpha}\left(\mathbb{R}^{d}\right)$. Similarly as in the case of classical nonlinear heat equation (1.1) with $\alpha=2$, cf. [40, Theorems 20.2, 20.6, 20.15], one may expect 
better decay rate under stronger assumptions on the regularity of the initial data even for somewhat bigger size of data than in the proof of Theorem 2.6.

\section{Asymptotic behavior of solutions below the singular solution}

We consider in this section behavior of solutions close to the singular solution $u_{\infty}$ but lying below it. We use the same approach as in [37] for classical nonlinear heat equation.

Introducing a new variable $w(x, t)=u_{\infty}(x)-u(x, t)$, where $u=u(x, t)$ is a solution to (1.1)-(1.2) the considered problem takes the form

$$
\begin{gathered}
w_{t}=-(-\Delta)^{\alpha / 2} w+s(\alpha, d, p)^{p-1} p|x|^{-\alpha} w-\left(\left(u_{\infty}-w\right)^{p}-u_{\infty}^{p}+p u_{\infty}^{p-1} w\right) \\
w(x, 0)=w_{0}(x)
\end{gathered}
$$

where the constant $s(\alpha, d, p)$ is defined in formula (2.2). Note that the last term on the right-hand side of equation (3.1) is nonpositive, namely

$$
\left(u_{\infty}-w\right)^{p}-u_{\infty}^{p} \geqslant-p u_{\infty}^{p-1} w
$$

which is the direct consequence of the convexity of the function $f(s)=|s|^{p}$ on $(0, \infty)$ for $p \geqslant 1$. Indeed, since the graph of the function $f$ lies above all of its tangents, we have $f(s-h)-f(s) \geqslant-f^{\prime}(s) h$ for all $s$ and $h$ in $\mathbb{R}$.

The proofs of our results are based on the following elementary observation. If $w$ is a nonnegative solution of equation (3.1) with the initial condition $w_{0}(x) \geqslant 0$, then

$$
0 \leqslant w(x, t) \leqslant \mathrm{e}^{-t H} w_{0}(x)
$$

with the operator $H w=(-\Delta)^{\alpha / 2} w-s(\alpha, d, p) p|x|^{-\alpha} w$. Consequently, using the condition $0 \leqslant u_{0}(x) \leqslant u_{\infty}(x)$, and the just mentioned comparison principle we can write

$$
0 \leqslant u_{\infty}(x)-u(x, t) \leqslant \mathrm{e}^{-t H}\left(u_{\infty}(x)-u_{0}(x)\right)
$$

or, equivalently,

$$
u_{\infty}(x)-\mathrm{e}^{-t H}\left(u_{\infty}(x)-u_{0}(x)\right) \leqslant u(x, t) \leqslant u_{\infty}(x) .
$$

First, we concentrate on existence and properties of solutions to the linear initial value problem

$$
\begin{aligned}
w_{t} & =-(-\Delta)^{\alpha / 2} w+s(\alpha, d, p)^{p-1} p|x|^{-\alpha} w, \\
w(x, 0) & =w_{0}(x) .
\end{aligned}
$$

\subsection{Linear fractional equation with the Hardy potential}

In this section we recall from [17] the estimate from above of the fundamental solution of the equation $u_{t}=-(-\Delta)^{\alpha / 2} u+\kappa|x|^{-\alpha} u$ for moderate values of $\kappa$, i.e. $\kappa \leqslant \frac{(2 \pi)^{\alpha}}{c_{\alpha}}$ with the constant

$$
c_{\alpha}=\pi^{\alpha}\left[\Gamma\left(\frac{d-\alpha}{4}\right) / \Gamma\left(\frac{d+\alpha}{4}\right)\right]^{2}
$$


appearing in a fractional version of the Hardy inequality. Following those arguments, we define the weights $\varphi_{\sigma}(x, t) \in \mathcal{C}\left(\mathbb{R}^{d} \backslash\{0\}\right)$ as

$$
\varphi_{\sigma}(x, t)=1+t^{\sigma / \alpha}|x|^{-\sigma}
$$

where $\sigma \in(0, d-\alpha)$ satisfies the following equality

$$
-2^{\alpha}+\lambda \frac{\Gamma\left(\frac{\sigma}{2}\right) \Gamma\left(\frac{d}{2}-\frac{\sigma+\alpha}{2}\right)}{\Gamma\left(\frac{d}{2}-\frac{\sigma}{2}\right) \Gamma\left(\frac{\sigma+\alpha}{2}\right)}=0 .
$$

Theorem 3.1. Let $\alpha \in(0, d \wedge 2), H u=-(-\Delta)^{\alpha / 2} u+s(\alpha, d, p)^{p-1} p|x|^{-\alpha} u$. Assume that $0 \leqslant s(\alpha, d, p)^{p-1} p \leqslant \frac{(2 \pi)^{\alpha}}{c_{\alpha}}$ with $c_{\alpha}$ defined in (3.7).

The semigroup $\mathrm{e}^{-t H}$ of the linear operators generated by $H$ can be written as the integral operator with a kernel denoted by $\mathrm{e}^{-t H}(x, y)$, namely

$$
\mathrm{e}^{-t H} u_{0}(x)=\int_{\mathbb{R}^{n}} \mathrm{e}^{-t H}(x, y) u_{0}(y) \mathrm{d} y .
$$

Moreover, there exists a positive constant $C$ such that for all $t>0$ and all $x, y \in \mathbb{R}^{d} \backslash\{0\}$

$$
\begin{aligned}
0 & \leqslant \mathrm{e}^{-t H}(x, y) \leqslant C \varphi_{\sigma}(x, t) \varphi_{\sigma}(y, t) G_{\alpha}(x-y, t) \\
0 & \leqslant \mathrm{e}^{-t H}(x, y) \\
& \leqslant C\left(1+t^{\sigma / \alpha}|x|^{-\sigma}\right)\left(1+t^{\sigma / \alpha}|y|^{-\sigma}\right)\left(t^{-d / \alpha} \wedge \frac{t}{|x-y|^{d+\alpha}}\right),
\end{aligned}
$$

where the functions $\varphi_{\sigma}$ are defined in (3.8) and $G_{\alpha}(x-y, t)$ is the fractional heat kernel.

Remark 3.2. Another important exponent for $\alpha=2$ is the Joseph-Lundgren exponent $p_{J L}=\frac{d-2 \sqrt{d-1}}{d-4-2 \sqrt{d-1}}$ which plays a crucial role in the study of stability of solutions to the classical nonlinear heat equation (see [37] and references therein). The analogue of this exponent for $\alpha \in(0,2)$ is the critical value of $p$ for which the assumption $s(\alpha, d, p)^{p-1} p \leqslant \frac{(2 \pi)^{\alpha}}{c_{\alpha}}$ is fulfilled. Observe that the assumption

$$
s(\alpha, d, p)^{p-1} p \leqslant \frac{(2 \pi)^{\alpha}}{c_{\alpha}}
$$

is satisfied for certain $d$ (large) and $p$ (close to $\frac{d}{d-\alpha}>1$ ). This follows from the asymptotics of the expression $s(\alpha, d, p)^{p-1} p$ for $p \searrow \frac{d}{d-\alpha}$. Indeed,

$$
\lim _{p \searrow \frac{d}{d-\alpha}} s(\alpha, d, p)^{p-1} p=0,
$$

thus there exists $p>\frac{d}{d-\alpha}$ satisfying inequality (3.11). Note that the exponent $p=\frac{d+\alpha}{d-\alpha}>1$ does not satisfy assumption (3.11) since according to [16, Remark $3]$ $s\left(\alpha, d, \frac{d+\alpha}{d-\alpha}\right)^{\frac{2 \alpha}{d-\alpha}}=\frac{(2 \pi)^{\alpha}}{c_{\alpha}}$.

The following theorem is the consequence of the estimates stated in formulas (3.10). 
Theorem 3.3. Let the assumptions of Theorem 3.1 be valid and $\sigma \in(0, d-\alpha)$ satisfy equation (3.9). Assume that $p>1+\frac{\alpha}{d-\sigma}$. Suppose that there exist $b>0$ and $\ell \in\left(\frac{\alpha}{p-1}, d-\sigma\right)$ such that if a nonnegative function $w_{0}$ satisfies

$$
\begin{aligned}
& w_{0}(x) \leqslant b|x|^{-\frac{\alpha}{p-1}} \quad \text { for } \quad|x| \leqslant 1 \\
& w_{0}(x) \leqslant b|x|^{-\ell} \quad \text { for } \quad|x| \geqslant 1
\end{aligned}
$$

then

$$
\sup _{x \in \mathbb{R}^{d}} \varphi_{\sigma}^{-1}(x, t)\left|\mathrm{e}^{-t H} w_{0}(x)\right| \leqslant C t^{-\frac{\ell}{2}}
$$

holds for a constant $C=C(b)>0$ and all $t \geqslant 1$.

Proof. First, for every fixed $x \in \mathbb{R}^{d}$, we apply the estimate of the kernel $\mathrm{e}^{-t H}$ in Theorem 3.1 in the following way

$$
\varphi_{\sigma}^{-1}(x, t)\left|\mathrm{e}^{-t H} w_{0}(x)\right| \leqslant C \int_{\mathbb{R}^{d}} G_{\alpha}(x-y, t) \varphi_{\sigma}(y, t) w_{0}(y) \mathrm{d} y .
$$

Next, we split the integral on the right-hand side into three parts $I_{1}(x, t)$, $I_{2}(x, t)$ and $I_{3}(x, t)$ according to the definition of the weights $\varphi_{\sigma}$ and the assumptions on the function $w_{0}$. Let us begin with $I_{1}(x, t)$

$$
\begin{aligned}
I_{1}(x, t) & \equiv C \int_{|y| \leqslant 1} G_{\alpha}(x-y, t) \varphi_{\sigma}(y, t) w_{0}(y) \mathrm{d} y \\
& \leqslant C(b) t^{\sigma / \alpha} \int_{|y| \leqslant 1} G_{\alpha}(x-y, t)|y|^{-\sigma-\frac{\alpha}{p-1}} \mathrm{~d} y \leqslant C(b) t^{-(d-\sigma) / \alpha},
\end{aligned}
$$

because $G_{\alpha}(x-y, t)$ is bounded by $C t^{-d / \alpha}$ and the function $|y|^{-\sigma-\frac{\alpha}{p-1}}$ is integrable for $|y| \leqslant 1$ if $p>1+\frac{\alpha}{d-\sigma}$. Here, the constant $C(b)$ depends on $b$.

We use the same argument to deal with

$$
\begin{aligned}
I_{2}(x, t) & \equiv C \int_{1 \leqslant|y| \leqslant t^{1 / \alpha}} G_{\alpha}(x-y, t) \varphi_{\sigma}(y, t) w_{0}(y) \mathrm{d} y \\
& \leqslant C(b) t^{\sigma / \alpha} \int_{1 \leqslant|y| \leqslant t^{1 / \alpha}} G_{\alpha}(x-y, t)|y|^{-\sigma-\ell} \mathrm{d} y \\
& \leqslant C(b) t^{\sigma-d / \alpha} \int_{1 \leqslant|y| \leqslant t^{1 / \alpha}}|y|^{-\sigma-\ell} \mathrm{d} y \\
& \leqslant C(b) t^{-\ell / \alpha}+C(b) t^{-(d-\sigma) / \alpha} .
\end{aligned}
$$

Finally, we estimate

$$
\begin{aligned}
I_{3}(x, t) & \equiv C \int_{|y| \geqslant t^{1 / \alpha}} G_{\alpha}(x-y, t) \varphi_{\sigma}(y, t) w_{0}(y) \mathrm{d} y \\
& \leqslant C(b) \int_{|y| \geqslant t^{1 / \alpha}} G_{\alpha}(x-y, t)|y|^{-\ell} \mathrm{d} y \leqslant C(b) t^{-\ell / \alpha},
\end{aligned}
$$


using the inequality $1 \leqslant\left(\frac{t^{1 / \alpha}}{|y|}\right)^{\ell}$ for $|y| \geqslant t^{1 / \alpha}$ and the identity $\int_{\mathbb{R}^{d}} G_{\alpha}(x-$ $y, t) \mathrm{d} y=1$ for $t>0, x \in \mathbb{R}^{d}$. Since $\ell \in\left(\frac{\alpha}{p-1}, d-\sigma\right)$, the proof of (3.12) is completed.

Theorem 3.4. Assume that $|\cdot|{ }^{\sigma} w_{0} \in L^{\infty}\left(\mathbb{R}^{d}\right)$ and $\lim _{|x| \rightarrow \infty}|x|^{\sigma} w_{0}(x)=0$, where $\sigma \in(0, d-\alpha)$ satisfies equation (3.9). Then

$$
\lim _{t \rightarrow \infty} t^{\sigma / \alpha} \sup _{x \in \mathbb{R}^{d}} \varphi_{\sigma}^{-1}(x, t)\left|\mathrm{e}^{-t H} w_{0}(x)\right|=0 .
$$

Proof. For every fixed $x \in \mathbb{R}^{d}$ we use the estimate from Theorem 3.1 as follows

$$
\varphi_{\sigma}^{-1}(x, t)\left|\mathrm{e}^{-t H} w_{0}(x)\right| \leqslant C \int_{\mathbb{R}^{d}} G_{\alpha}(x-y, t) \varphi_{\sigma}(y, t) w_{0}(y) \mathrm{d} y .
$$

We decompose the integral on the right-hand side according to the definition of $\varphi_{\sigma}$ and we estimate each term separately. Substituting $y=z t^{1 / \alpha}$ and using the fact that $\varphi_{\sigma}(x, t) \leqslant 2 t^{\sigma / \alpha}|x|^{-\sigma}$ if $|x| \leqslant t^{1 / \alpha}$ we obtain

$$
\begin{aligned}
I_{1}(x, t) & \equiv C \int_{|y| \leqslant t^{1 / \alpha}} G_{\alpha}(x-y, t)\left(\frac{t^{1 / \alpha}}{|y|}\right)^{\sigma} w_{0}(y) \mathrm{d} y \\
& =C t^{-\sigma / \alpha} \int_{|z| \leqslant 1} G\left(\frac{x}{t^{1 / \alpha}}-z, t\right)|z|^{-2 \sigma}\left|t^{1 / \alpha} z\right|^{\sigma} w_{0}\left(t^{1 / \alpha} z\right) \mathrm{d} z .
\end{aligned}
$$

Hence,

$$
t^{\sigma / \alpha} \sup _{x \in \mathbb{R}^{d}} I_{1}(x, t) \rightarrow 0 \quad \text { as } \quad t \rightarrow \infty
$$

follows by the Lebesgue dominated convergence theorem, because $G\left(\frac{x}{t^{1 / \alpha}}-z, 1\right)$ is bounded and the function $|z|^{-2 \sigma}$ is integrable for $|z| \leqslant 1$. By the assumption imposed on $w_{0}$, given $\varepsilon>0$ we may choose $t$ so large that

$$
\sup _{|y| \geqslant \sqrt{t}}|y|^{\sigma} w_{0}(y)<\varepsilon \text {. }
$$

Now, using the inequality $1 \leqslant\left(\frac{t^{1 / \alpha}}{|y|}\right)^{\sigma}$ for $|y| \geqslant t^{1 / \alpha}$, we obtain

$$
\begin{aligned}
I_{2}(x, t) & \equiv \int_{|y| \geqslant t^{1 / \alpha}} G_{\alpha}(x-y, t) w_{0}(y) \mathrm{d} y \\
& \leqslant t^{-\sigma / \alpha} \int_{|y| \geqslant t^{1 / \alpha}} G_{\alpha}(x-y, t)|y|^{\sigma} w_{0}(y) \mathrm{d} y \\
& \leqslant \varepsilon t^{-\sigma / \alpha} \int_{|y| \geqslant t^{1 / \alpha}} G_{\alpha}(x-y, t) \mathrm{d} y .
\end{aligned}
$$

Since $\int_{\mathbb{R}^{d}} G(x-y, t) \mathrm{d} y=1$ for all $t>0, x \in \mathbb{R}^{d}$ and since $\varepsilon>0$ is arbitrary, we get

$$
t^{\sigma / \alpha} \sup _{x \in \mathbb{R}^{d}} I_{2}(x, t) \rightarrow 0 \quad \text { as } \quad t \rightarrow \infty
$$


Let us define the weighted $L^{q}\left(\mathbb{R}^{d}\right)$-norm as follows

$$
\|f\|_{q, \varphi_{\sigma}(t)}=\left(\int_{\mathbb{R}^{d}}\left|f(x) \varphi_{\sigma}^{-1}(x, t)\right|^{q} \varphi_{\sigma}^{2}(x, t) \mathrm{d} x\right)^{\frac{1}{q}} \quad \text { for each } \quad 1 \leqslant q<\infty,
$$

and

$$
\|f\|_{\infty, \varphi_{\sigma}(t)}=\sup _{x \in \mathbb{R}^{d}} \varphi_{\sigma}^{-1}(x, t)|f(x)| \text { for } \quad q=\infty .
$$

Note, that in particular for $q=2$, the norm $\|\cdot\|_{2, \varphi_{\sigma}(t)}$ coincides with the usual $L^{2}$-norm on $\mathbb{R}^{d}$.

Proposition 3.5. (hypercontractivity estimates) Suppose that $1 \leqslant q \leqslant \infty$. Then the following inequality holds true

$$
\left\|\mathrm{e}^{-t H} w_{0}\right\|_{q, \varphi_{\sigma}(t)} \leqslant C t^{-\frac{d}{\alpha}\left(\frac{1}{r}-\frac{1}{q}\right)}\left\|w_{0}\right\|_{r, \varphi_{\sigma}(t)}
$$

for every $1 \leqslant r \leqslant q \leqslant \infty$ and all $t>0$, where weights $\varphi_{\sigma}$ are defined in (3.8).

Proof. Using estimates of the kernel $\mathrm{e}^{-t H}$ we get

$$
\begin{aligned}
& \left\|\varphi_{\sigma}^{-1} e^{-t H} \varphi_{\sigma} w_{0}\right\|_{p, \varphi_{\sigma}(t)}^{p} \\
& \quad=\int_{\mathbb{R}^{d}} \varphi_{\sigma}^{-p}(x, t)\left|e^{-t H} w_{0}(x) \varphi_{\sigma}(x, t)\right|^{p} \varphi_{\sigma}^{2}(x, t) \mathrm{d} x \\
& \quad=\int_{\mathbb{R}^{d}} \varphi_{\sigma}^{-p+2}(x, t)\left|\int_{\mathbb{R}^{d}} e^{-t H}(x, y) \varphi_{\sigma}^{2}(y, t) w_{0}(y) \mathrm{d} y\right|^{p} \mathrm{~d} x \\
& \leqslant C \int_{\mathbb{R}^{d}} \varphi_{\sigma}^{-p+2}(x, t)\left|\int_{\mathbb{R}^{d}} G_{\alpha}(x-y, t) \varphi_{\sigma}(x, t) \varphi_{\sigma}^{2}(y, t) w_{0}(y) \mathrm{d} y\right|^{p} \mathrm{~d} x \\
& =C \int_{\mathbb{R}^{d}} \varphi_{\sigma}^{2}(x, t)\left|\int_{\mathbb{R}^{d}} G_{\alpha}(x-y, t) \varphi_{\sigma}^{2}(y, t) w_{0}(y) \mathrm{d} y\right|^{p} \mathrm{~d} x .
\end{aligned}
$$

Observe that the weights are bounded by

$$
\begin{aligned}
& \varphi_{\sigma}(x, t) \leqslant 1 \quad \text { if }|x| \geqslant t^{1 / \alpha}, \\
& \varphi_{\sigma}(x, t) \leqslant 2 t^{\sigma / \alpha}|x|^{-\sigma} \text { if }|x| \leqslant t^{1 / \alpha} .
\end{aligned}
$$

We split the integral into two terms

$$
\begin{aligned}
& I_{1}=\left.C \int_{|x| \geqslant t^{1 / \alpha}} \varphi_{\sigma}^{2}(x, t) \int_{\mathbb{R}^{d}} G_{\alpha}(x-y, t) \varphi_{\sigma}^{2}(y, t) w_{0}(y) \mathrm{d} y\right|^{p} \mathrm{~d} x, \\
& I_{2}=C \int_{|x| \leqslant t^{1 / \alpha}} \varphi_{\sigma}^{2}(x, t)\left|\int_{\mathbb{R}^{d}} G_{\alpha}(x-y, t) \varphi_{\sigma}^{2}(y, t) w_{0}(y) \mathrm{d} y\right|^{p} \mathrm{~d} x,
\end{aligned}
$$

and applying (3.15) we get

$$
\begin{aligned}
I_{1} & \leqslant C \int_{|x| \geqslant t^{1 / \alpha}}\left|\int_{\mathbb{R}^{d}} G_{\alpha}(x-y, t) \varphi_{\sigma}^{2}(y, t) w_{0}(y) \mathrm{d} y\right|^{p} \mathrm{~d} x \\
& \leqslant \int_{\mathbb{R}^{d}}\left|\int_{\mathbb{R}^{d}} G_{\alpha}(x-y, t) \varphi_{\sigma}^{2}(y, t) w_{0}(y) \mathrm{d} y\right|^{p} \mathrm{~d} x \\
& =C\left\|G_{\alpha}(t) w_{0} \varphi_{\sigma}^{2}\right\|_{p}^{p} \leqslant C t^{-d / \alpha\left(\frac{1}{q}-\frac{1}{p}\right) p}\left\|w_{0} \varphi_{\sigma}^{2}\right\|_{q}^{p}
\end{aligned}
$$


using estimates of the semigroup generated by the fractional Laplacian. Moreover, applying the Young inequality, and estimates of this semigroup we arrive at

$$
\begin{aligned}
I_{2} & =C \int_{|x| \leqslant t^{1 / \alpha}} t^{\sigma / \alpha}|x|^{-2 \sigma}\left|\int_{\mathbb{R}^{d}} G_{\alpha}(x-y, t) \varphi_{\sigma}^{2}(y, t) w_{0}(y) \mathrm{d} y\right|^{p} \mathrm{~d} x \\
& \leqslant C t^{\sigma / \alpha} \int_{|x| \leqslant t^{1 / \alpha}}|x|^{-2 \sigma}\left\|G_{\alpha}(t) w_{0} \varphi_{\sigma}^{2}\right\|_{\infty}^{p} \mathrm{~d} x \\
& \leqslant C t^{\sigma / \alpha} \int_{|x| \leqslant t^{1 / \alpha}}|x|^{-2 \sigma}\left\|G_{\alpha}(t)\right\|_{\frac{q}{q-1}}^{p}\left\|w_{0} \varphi_{\sigma}^{2}\right\|_{q}^{p} \mathrm{~d} x \\
& \leqslant C t^{2 \sigma / \alpha-\frac{d p}{\alpha q}}\left\|w_{0} \varphi_{\sigma}^{2}\right\|_{q}^{p} \int_{|x| \leqslant t^{1 / \alpha}}|x|^{-2 \sigma} \mathrm{d} x \\
& =C t^{-\frac{d}{\alpha}\left(\frac{1}{q}-\frac{1}{p}\right) p}\left\|w_{0} \varphi_{\sigma}^{2}\right\|_{q}^{p},
\end{aligned}
$$

which completes the proof.

\subsection{Nonlinear equation}

Now we are in a position to state the result on convergence of solutions towards the singular steady state.

Theorem 3.6. Let $u=u(x, t)$ be a solution to problem (1.1)-(1.2) constructed in Theorem 2.6 with exponent $p$ satisfying assumption (3.11) and $\sigma \in(0, d-\alpha)$ fulfill equation (3.9). Assume that there exist constants $b>0$ and $\ell \in(\sigma, d-\sigma)$ such that

$$
u_{\infty}(x)-b|x|^{-\ell} \leqslant u_{0}(x)
$$

for all $|x| \geqslant 1$. Then

$$
\sup _{|x| \leqslant t^{1 / \alpha}}|x|^{\sigma}\left(u_{\infty}(x)-u(x, t)\right) \leqslant C t^{-\ell-\sigma / \alpha}
$$

and

$$
\sup _{|x| \geqslant t^{1 / \alpha}}\left(u_{\infty}(x)-u(x, t)\right) \leqslant C t^{-\ell / \alpha} .
$$

hold for a constant $C>0$ and all $t \geqslant 1$.

Remark 3.7. For the classical nonlinear heat equation Poláčik and Yanagida [39, Th. 6.1] proved a pointwise convergence of solutions to the singular steady state for $x \in \mathbb{R}^{d} \backslash\{0\}$. Moreover, Fila and Winkler [24] showed the uniform convergence of solutions to the singular one on $\mathbb{R}^{d} \backslash B_{r}(0)$, where $B_{r}(0)$ is the ball centered at the origin with the radius $r>0$.

Proof. It suffices to use inequality (3.3) and to estimate its right-hand side by Theorem 3.3.

We can improve Theorem 3.6 for the limit exponent $\ell=\sigma$ as follows. 
Theorem 3.8. Let $u=u(x, t)$ be a solution to problem (1.1)-(1.2) constructed in Theorem 2.6 with exponent $p$ satisfying assumption (3.11), and $\sigma \in(0, d-\alpha)$ fulfill equation (3.9). Suppose that there exists a constant $b>0$ such that

$$
u_{\infty}(x)-b|x|^{-\sigma} \leqslant u_{0}(x),
$$

and, moreover,

$$
\lim _{|x| \rightarrow \infty}|x|^{\sigma}\left(u_{\infty}(x)-u_{0}(x)\right)=0
$$

Then the relations

$$
\lim _{t \rightarrow \infty} \sup _{|x| \leqslant t^{1 / \alpha}}|x|^{\sigma}\left(u_{\infty}(x)-u(x, t)\right)=0
$$

and

$$
\lim _{t \rightarrow \infty} t^{\sigma / \alpha} \sup _{|x| \geqslant t^{1 / \alpha}}\left(u_{\infty}(x)-u(x, t)\right)=0
$$

hold.

Remark 3.9. A similar result for the classical case, namely for $\alpha=2$, can be found in [23-26], where authors proved estimates from below of the $L^{\infty}$-norm of solutions using matched asymptotics.

Proof. As in the proof of Theorem 3.6, it is sufficient to use (3.3) together with Theorem 3.4, substituting $w_{0}(x)=u_{\infty}(x)-u_{0}(x)$.

Corollary 3.10. Under the assumptions of Theorems 3.6 and 3.8, respectively if, moreover, $b$ is sufficiently small, we obtain

$$
\|u(\cdot, t)\|_{\infty} \geqslant C t^{\frac{\ell-\sigma}{\sigma(p-1)-\alpha}} \quad \text { if } \quad \ell \in(\sigma, d-\sigma)
$$

for a constant $C>0$ and all $t \geqslant 1$, and

$$
\lim _{t \rightarrow \infty}\|u(\cdot, t)\|_{\infty}=\infty \quad \text { if } \quad \ell=\sigma .
$$

Proof. Since we have inequality (3.4), it suffices to prove that

$$
\sup _{x \in \mathbb{R}^{d}}\left(u_{\infty}(x)-\mathrm{e}^{-t H} w_{0}(x)\right) \geqslant C(b) t^{\frac{\ell-\sigma}{\sigma(p-1)-\alpha}}
$$

for $w_{0}=u_{\infty}-u_{0}$. Hence, inequality (3.12) in Theorem 3.3 enables us to write

$$
u_{\infty}(x)-\mathrm{e}^{-t H}\left(u_{\infty}(x)-u_{0}(x)\right) \geqslant u_{\infty}(x)-C(b) \varphi_{\sigma}(x, t) t^{-\ell / \alpha}
$$

for all $x \in \mathbb{R}^{d} \backslash\{0\}$ and $t>0$. Next, using the explicit form of the weights $\varphi_{\sigma}$, we define the function

$$
\begin{aligned}
F(|x|, t) & =u_{\infty}(|x|)-C(b) \varphi_{\sigma}(x, t) t^{-\ell / \alpha} \\
& = \begin{cases}s(\alpha, d, p)^{p-1} p|x|^{-\frac{\alpha}{p-1}}-C(b) t^{(\sigma-\ell) / \alpha}|x|^{-\sigma} & \text { for }|x| \leqslant t^{1 / \alpha}, \\
s(\alpha, d, p)^{p-1} p|x|^{-\frac{\alpha}{p-1}}-C(b) t^{-\ell / \alpha} & \text { for }|x|>t^{1 / \alpha} .\end{cases}
\end{aligned}
$$

An easy computation shows that the function $F$ attains its maximum at

$$
|x|=C(b) t^{(\sigma-\ell) / \alpha \frac{p-1}{\sigma(p-1)-\alpha}},
$$


and this is equal to

$$
\max _{x \in \mathbb{R}^{d}} F(|x|, t)=C(b) t^{\frac{\ell-\sigma}{\sigma(p-1)-\alpha}}
$$

for some constant $C(b)>0$. Hence, we get (3.18).

To obtain (3.19), we use the result from Theorem 3.4. It follows from (3.13) that for every $\varepsilon>0$ there exists $T>0$ such that

$$
\left|\mathrm{e}^{-t H} w_{0}(x)\right| \leqslant \varepsilon \varphi_{\sigma}(x, t) t^{-\sigma / \alpha}
$$

for all $x \in \mathbb{R}^{d} \backslash\{0\}$ and $t>T$. Hence, by (3.4), we have

$$
u_{\infty}(x)-\mathrm{e}^{-t H}\left(u_{\infty}(x)-u_{0}(x)\right) \geqslant u_{\infty}(x)-C \varepsilon \varphi_{\sigma}(x, t) t^{-\sigma / \alpha} .
$$

Now, using again the explicit form of the weights $\varphi_{\sigma}$, we consider the function

$$
\begin{aligned}
G(|x|, t) & =v_{\infty}(|x|)-C(b) \varphi_{\sigma}(x, t) t^{-\sigma / \alpha} \\
& = \begin{cases}s(\alpha, d, p)^{p-1} p|x|^{-\frac{\alpha}{p-1}}-\varepsilon|x|^{-\sigma} & \text { for }|x| \leqslant t^{1 / \alpha} \\
s(\alpha, d, p)^{p-1} p|x|^{-\frac{\alpha}{p-1}}-\varepsilon t^{-\sigma / \alpha} & \text { for }|x|>t^{1 / \alpha}\end{cases}
\end{aligned}
$$

Elementary computations give us that the function $G$ attains its maximum at

$$
|x|=c \varepsilon^{-\frac{p-1}{\sigma(p-1)-\alpha}}
$$

and

$$
\max _{x \in \mathbb{R}^{d}} G(|x|, t)=C \varepsilon^{-\frac{\alpha}{\sigma(p-1)-\alpha}}
$$

for some constant $C \geqslant 0$. Since $\sigma>\frac{\alpha}{(p-1)}$, we see that the maximum of the function $G$ diverges to infinity if $\varepsilon$ tends to zero. This completes the proof of (3.19).

Our next goal is to prove the asymptotic stability of the singular solution $u_{\infty}$ in the Lebesgue space $L^{2}\left(\mathbb{R}^{d}\right)$.

Theorem 3.11. Let $u=u(x, t)$ be a solution to problem (1.1)-(1.2) with exponent $p$ satisfying assumption (3.11) and $\sigma \in(0, d-\alpha)$ fulfill equation (3.9).

i) Suppose that $u_{\infty}-u_{0} \in L^{1}\left(\mathbb{R}^{d}\right)$ and $|\cdot|^{-\sigma}\left(u_{\infty}-u_{0}\right) \in L^{1}\left(\mathbb{R}^{d}\right)$. Then

$$
\left\|u_{\infty}-u(t)\right\|_{2} \leqslant C t^{-\frac{d}{2 \alpha}}\left\|u_{\infty}-u_{0}\right\|_{1}+C t^{-\frac{d-2 \sigma}{2 \alpha}}\left\||\cdot|^{-\sigma}\left(u_{\infty}-u_{0}\right)\right\|_{1} .
$$

ii) Suppose that $u_{\infty}-u_{0} \in L^{2}\left(\mathbb{R}^{d}\right)$. Then

$$
\lim _{t \rightarrow \infty}\left\|u_{\infty}-u(t)\right\|_{2}=0 .
$$

Proof of Theorem 3.11 (i). According to estimates (3.3) it is enough to estimate the $L^{2}$-norm of the expression $\mathrm{e}^{-t H} w_{0}$ for every $w_{0}$ satisfying two conditions: $w_{0} \in L^{1}\left(\mathbb{R}^{d}\right)$ and $|\cdot|^{-\sigma} w_{0} \in L^{1}\left(\mathbb{R}^{d}\right)$. Applying (3.14) with $q=2, r=1$, and using the definition of the functions $\varphi_{\sigma}(x, t)$, we may write 


$$
\begin{aligned}
\left\|\mathrm{e}^{-t H} w_{0}\right\|_{2} & \leqslant C t^{-\frac{d}{2 \alpha}}\left\|w_{0}\right\|_{1, \varphi_{\sigma}(t)}=C t^{-\frac{d-2 \sigma}{2 \alpha}} \int_{|x| \leqslant t^{1 / \alpha}} w_{0}(x)|x|^{-\sigma} \mathrm{d} x \\
& +C t^{-\frac{d}{2 \alpha}} \int_{|x| \geqslant t^{1 / \alpha}} w_{0}(x) \mathrm{d} x \leqslant C t^{-\frac{d-2 \sigma}{2 \alpha}}\left\|w_{0}|\cdot|^{-\sigma}\right\|_{1}+C t^{-\frac{d}{2 \alpha}}\left\|w_{0}\right\|_{1} .
\end{aligned}
$$

This establishes formula (3.20).

Proof of Theorem 3.11 (ii). Again, by inequalities (3.3), we only need to show that

$$
\lim _{t \rightarrow \infty}\left\|\mathrm{e}^{-t H} w_{0}\right\|_{2}=0
$$

for each $w_{0} \in L^{2}\left(\mathbb{R}^{d}\right)$. Hence, for every $\varepsilon>0$ we choose $\psi \in C_{c}^{\infty}\left(\mathbb{R}^{d}\right)$ such that $\left\|w_{0}-\psi\right\|_{2}<\varepsilon$. Using the triangle inequality first and next estimate (3.14) with $q=2$ and $r=2$, we obtain

$$
\begin{aligned}
\left\|\mathrm{e}^{-t H} w_{0}\right\|_{2} & \leqslant\left\|\mathrm{e}^{-t H}\left(w_{0}-\psi\right)\right\|_{2}+\left\|\mathrm{e}^{-t H} \psi\right\|_{2} \\
& \leqslant C \varepsilon+\left\|\mathrm{e}^{-t H} \psi\right\|_{2} .
\end{aligned}
$$

Since the second term on the right-hand side converges to zero as $t \rightarrow \infty$ by the first part of Theorem 3.11, we get

$$
\limsup _{t \rightarrow \infty}\left\|\mathrm{e}^{-t H} w_{0}\right\|_{2} \leqslant C \varepsilon \text {. }
$$

This completes the proof of Theorem 3.11 ii), because $\varepsilon>0$ can be arbitrarily small.

\subsection{Decay of solutions}

We prove an asymptotic result for solutions considered in Theorem 2.6

Theorem 3.12. Let $u$ be a solution of problem (1.1)-(1.2) with $u_{0} \in L_{\varphi_{\sigma}(t)}^{1}\left(\mathbb{R}^{d}\right)$ satisfying the assumptions of Theorem 2.6. Then

$$
\lim _{t \rightarrow \infty}\|u(t)\|_{q, \varphi_{\sigma}(t)}=0
$$

for each $1 \leqslant q \leqslant \infty$ holds.

Proof. By Proposition 2.7 we have $u(x, t)<\delta u_{\infty}(x)$ for $t>0$, hence

$$
u_{t}=-(-\Delta)^{\alpha / 2} u+|u|^{p-1} u<-(-\Delta)^{\alpha / 2} u+(\delta s(\alpha, d, p))^{p-1}|x|^{-\alpha} u .
$$

If $(\delta s(\alpha, d, p))^{p-1} \leqslant \frac{(2 \pi)^{\alpha}}{c_{\alpha}}$, where $c_{\alpha}$ is defined in (3.7), we get the estimate

$$
u(x, t) \leqslant e^{-t H_{\delta}} u_{0}(x),
$$


where $H_{\delta} u=-(-\Delta)^{\alpha / 2} u+(\delta s(\alpha, d, p))^{p-1}|x|^{-\alpha} u$. Using Proposition 3.5 for $1 \leqslant q \leqslant \infty$ and $r=1$ we obtain

$$
\|u(t)\|_{q, \varphi_{\sigma}(t)} \leqslant C t^{-\frac{d}{\alpha}\left(1-\frac{1}{q}\right)}\left\|u_{0}\right\|_{1, \varphi_{\sigma}(t)},
$$

which completes the proof of Theorem 3.12.

\section{Complements and comments}

A sufficient condition for blowup of solutions of equation (1.1) with $p>1+\frac{\alpha}{d}$

$$
T^{\frac{1}{p-1}}\left\|\mathrm{e}^{-t(-\Delta)^{\alpha / 2}} u_{0}\right\|_{\infty}>C_{\alpha, d, p}
$$

derived in [43] has been interpreted in [7] as

$$
\left\|u_{0}\right\|_{M^{d(p-1) / \alpha}}>m_{\alpha, d, p}
$$

for some $m_{\alpha, d, p}>0$. Indeed, we have equivalence

$$
\sup _{t>0} t^{\gamma}\left\|\mathrm{e}^{-t(-\Delta)^{\alpha / 2}} u\right\|_{\infty}<\infty \text { if and only if } u \in B_{\infty, \infty}^{-\gamma \alpha}\left(\mathbb{R}^{d}\right)
$$

where $B_{\infty, \infty}^{-\kappa}$ is the homogeneous Besov space of order $-\kappa<0$. The above condition (4.3) is for $u \geq 0$ equivalent to $u \in M^{\frac{d}{\alpha \gamma}}\left(\mathbb{R}^{d}\right)$, the Morrey space of order $\frac{d}{\alpha \gamma}$, [33, Prop. 2B)] for $\alpha=2$ and a slight modification of [34, Sec. 4, proof of Prop. 2] for $\alpha \in(0,2)$.

These are counterparts of results in [6, Remark 7, Theorem 2] for the classical nonlinear heat equation. Together with results of Sect. 2, this leads to the following partial dichotomy result, similarly as was in [6, Corollary $11]$

Corollary 4.1. (dichotomy) There exist two positive constants $c(\alpha, d, p)$ and $C(\alpha, d, p)$ such that if $p>1+\frac{\alpha}{d}$ then

(i) $\left|u_{0}\right|_{M_{q}^{d(p-1) / \alpha}}<c(\alpha, d, p)$ for some $q>1, q<\frac{d(p-1)}{\alpha}$, implies that problem (1.1)-(1.2) has a global in time, smooth solution satisfying the time decay estimate $\|u(t)\|_{\infty}=\mathcal{O}\left(t^{-1 /(p-1)}\right)$.

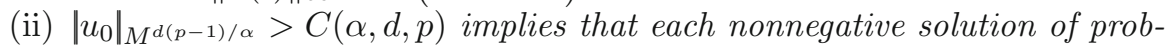
lem (1.1)-(1.2) blows up in a finite time.

Of course, there are many interesting behaviors of solutions (and still open questions) for the initial data of intermediate size satisfying

$$
c(\alpha, d, p) \leqslant \|\left. u_{0}\right|_{M^{d(p-1) / \alpha} \leqslant C(\alpha, d, p),}
$$

and/or suitable pointwise estimates comparing the initial condition $u_{0}$ with the singular solution $u_{\infty}$.

It is of interest to compare these constants $c(\alpha, d, p)$ and $C(\alpha, d, p)$ with the Morrey space norm $\left\|u_{\infty}\right\|_{M^{d(p-1) / \alpha}}$ of the singular stationary solution $u_{\infty}>$ 0 of (1.1) in (2.1)-(2.2).

Sufficient conditions for local-in-time existence of solutions are intimately connected with the problem of initial traces, i.e. a characterization of $u_{0}$ 
such that $u(t)$ tends to $u_{0}$ weakly as $t \rightarrow 0$ for a given nonnegative local solution of equation (1.1). Conditions on such $u_{0}$ 's, roughly speaking, mean that local singularities are weaker than a multiple of $|x|^{-\alpha /(p-1)}$ (or $\lim _{x \rightarrow x_{0}}\left|x-x_{0}\right|^{\alpha /(p-1)} u_{0}(x) \leqslant J(\alpha, d, p)$ for a universal constant $J(\alpha, d, p)>$ 0 ) so that the size of $u_{0}$ in $M_{\mathrm{loc}}^{d(p-1) / \alpha}\left(\mathbb{R}^{d}\right)$ is universally bounded.

Remark 4.2. (initial traces) General results on the existence of initial traces (i.e. $u_{0}$ 's) for arbitrary nonnegative weak solutions of equation (1.1) $(u=u(t)$ defined on $(0, T)$ ) can be inferred from $[2,3]$ using local moments like those in [13] with the weight functions $\left(1-|x|^{2}\right)_{+}^{1+\alpha / 2}$ used in the analysis of nonlocal problems of chemotaxis. In particular, estimates analogous to those in $[2$, (1.4), Prop. 4.3 on p. 380] show that a necessary condition for the existence of a local in time solution reads: $u_{0} \in M_{\mathrm{loc}}^{d(p-1) / \alpha}\left(\mathbb{R}^{d}\right)$, and each nonnegative solution satisfies $u(t) \in M_{\mathrm{loc}}^{d(p-1) / \alpha}\left(\mathbb{R}^{d}\right)$ uniformly on $(0, T)$. Here, $u_{0} \in$ $M_{\mathrm{loc}}^{d(p-1) / \alpha}\left(\mathbb{R}^{d}\right)$ means: $\lim \sup _{R \rightarrow 0, x \in \mathbb{R}^{d}} R^{\alpha /(p-1)-d} \int_{\{|y-x|<R\}}\left|u_{0}(y)\right| \mathrm{d} y<\infty$. Thus, $M^{d(p-1) / \alpha}\left(\mathbb{R}^{d}\right)$ is, in a sense, close to be the optimal space for the local in time solvability of problem (1.1)-(1.2) in the class of nonnegative solutions.

If in both (i) and (ii) of Corollary 4.1 on dichotomy there were a single functional norm $\bar{\ell}$ instead of those of $M_{q}^{d(p-1) / \alpha}\left(\mathbb{R}^{d}\right)$ with some $q>1$ and $M^{d(p-1) / \alpha}\left(\mathbb{R}^{d}\right)$ (both spaces are critical) this will be unique, up to equivalence (a personal communication of Philippe Souplet). Such a norm is called the dichotomy norm. Note that, however, problem (1.1)-(1.2) is not well posed in the critical space $M^{d(p-1) / \alpha}\left(\mathbb{R}^{d}\right)$, similarly to the case of radial solutions of the parabolic-elliptic Keller-Segel system studied in [13,33] with $M^{d / 2}\left(\mathbb{R}^{d}\right)$ data as well as for the fractional Keller-Segel system with $M^{d / \alpha}\left(\mathbb{R}^{d}\right)$ data in [8]. Namely, there is no continuity of solutions of the Cauchy problem (1.1)-(1.2) with respect to the initial data in this norm, see the following Remark 4.3. In view of the result in [20], one can barely expect that this $\bar{\ell}$ would be the norm in $M^{d(p-1) / \alpha}\left(\mathbb{R}^{d}\right)$ based solely on $L_{\text {loc }}^{1}$ properties of functions.

Remark 4.3. (solutions may depend discontinuously on the initial data in $\left.M^{d(p-1) / \alpha}\right)$ If the condition $\limsup _{R \rightarrow 0} R^{\alpha /(p-1)-d} \int_{\{|y-x|<R\}}\left|u_{0}(y)\right| \mathrm{d} y>$ $K(\alpha, d, p)$ is satisfied for some $x \in \mathbb{R}^{d}$ and a constant $K(\alpha, d, p) \geqslant C(\alpha, d, p)>$ 0 , then solutions are not continuous with respect to the initial data at $u_{0}$; in fact, the existence times of approximating solutions tend to 0 when the initial data are cut: $\mathbb{I}_{\left\{|y-x|>R_{n}\right\}} u_{0}, R_{n} \rightarrow 0$ as $n \rightarrow \infty$. This can be inferred from the sufficient condition for blowup and the estimate of the existence time for solutions, see analogous arguments in $[13,15]$.

Open Access. This article is distributed under the terms of the Creative Commons Attribution 4.0 International License (http://creativecommons.org/licenses/ by/4.0/), which permits unrestricted use, distribution, and reproduction in any medium, provided you give appropriate credit to the original author(s) and the 
source, provide a link to the Creative Commons license, and indicate if changes were made.

Publisher's Note Springer Nature remains neutral with regard to jurisdictional claims in published maps and institutional affiliations.

\section{References}

[1] Alfaro, M.: Fujita blow up phenomena and hair trigger effect: the role of dispersal tails. Ann. Inst. Henri Poincaré, Analyse non Linéaire 34, 1309-1327 (2017)

[2] Andreucci, D., DiBenedetto, E.: On the Cauchy problem and initial traces for a class of evolution equations with strongly nonlinear sources. Ann. Sc. Norm. Super. Pisa, Cl. Sci., IV. Ser. 18, 363-441 (1991)

[3] Baras, P., Pierre, M.: Critère d'existence de solutions positives pour des équations semi-linéaires non monotones. Ann. Inst. Henri Poincaré, Anal. non Linéaire 2, 185-212 (1985)

[4] Barrios, B., Peral, I., Soria, F., Valdinoci, E.: A Widder's type theorem for the heat equation with nonlocal diffusion. Arch. Ration. Mech. Anal. 213, 629-650 (2014)

[5] Biler, P.: The Cauchy problem and self-similar solutions for a nonlinear parabolic equation. Studia Math. 114, 181-205 (1995)

[6] Biler, P.: Blowup versus global in time existence of solutions for nonlinear heat equations, 1-13. Topol. Methods Nonlinear Anal. 52, 147-160 (2018)

[7] Biler, P.: Blowup of solutions for nonlinear nonlocal heat equations. pp. 1-12, Monatsh. Math., to appear; arXiv:1807.03569

[8] Biler, P.: Singularities of Solutions to Chemotaxis Systems, book in preparation, De Gruyter, Series in Mathematics and Life Sciences

[9] Biler, P., Funaki, T., Woyczyński, W.: Fractal Burgers equations. J. Differ. Equ. 148, 9-46 (1998)

[10] Biler, P., Imbert, C., Karch, G.: Nonlocal porous medium equation: Barenblatt profiles and other weak solutions. Arch. Ration. Mech. Anal. 215, 497-529 (2015)

[11] Biler, P., Karch, G., Woyczyński, W.A.: Critical nonlinearity exponent and selfsimilar asymptotics for Lévy conservation laws. Ann. Inst. Henri Poincaré, Anal. non Linéaire 18, 613-637 (2001)

[12] Biler, P., Karch, G., Woyczyński, W.A.: Asymptotics for conservation laws involving Lévy diffusion generators. Studia Math. 148, 171-192 (2001)

[13] Biler, P., Karch, G., Zienkiewicz, J.: Large global-in-time solutions to a nonlocal model of chemotaxis. Adv. Math. 330, 834-875 (2018)

[14] Biler, P., Karch, G., Pilarczyk, D.: Global radial solutions in classical KellerSegel chemotaxis model. pp. 1-20, submitted; arXiv:1807.02628 
[15] Biler, P., Zienkiewicz, J.: Blowing up radial solutions in the minimal KellerSegel chemotaxis model. J. Evol. Equ. 1-20 (2018). https://doi.org/10.1007/ s00028-018-0469-8

[16] Birkner, M., López-Mimbela, J.A., Wakolbinger, A.: Comparison results and steady states for the Fujita equation with fractional Laplacian. Ann. Inst. Henri Poincaré, Anal. non Linéaire 22, 83-97 (2005)

[17] Bogdan, K., Grzywny, T., Jakubowski, T., Pilarczyk, D.: Fractional Laplacian with Hardy potential. pp. 1-28; arXiv:1710.08378. Commun. Partial Differ. Equ. (2019). https://doi.org/10.1080/03605302.2018.1539102

[18] Bonforte, M., Sire, Y., Vázquez, J.L.: Optimal existence and uniqueness theory for the fractional heat equation. Nonlinear Anal., Theory Methods Appl., Ser. A, Theory Methods 153, 142-168 (2017)

[19] Burczak, J., Granero-Belinchón, R.: Global solutions for a supercritical driftdiffusion equation. Adv. Math. 295, 334-367 (2016)

[20] Celik, C., Zhou, Z.: No local $L^{1}$ solution for a nonlinear heat equation. Commun. Partial Differ. Equ. 28, 1807-1831 (2003)

[21] Constantin, P., Vicol, V.: Nonlinear maximum principles for dissipative linear nonlocal operators and applications. Geom. Funct. Anal. 22, 1289-1321 (2012)

[22] Córdoba, A., Córdoba, D.: A maximum principle applied to quasi-geostrophic equations. Commun. Math. Phys. 249, 511-528 (2004)

[23] Fila, M., King, J.R., Winkler, M., Yanagida, E.: Optimal lower bound of the grow-up rate for a supercritical parabolic equation. J. Differ. Equ. 228, 339-356 (2006)

[24] Fila, M., Winkler, M.: Rate of convergence to a singular steady state of a supercritical parabolic equation. J. Evol. Equ. 8, 673-692 (2008)

[25] Fila, M., Winkler, M., Yanagida, E.: Grow-up rate of solutions for a supercritical semilinear diffusion equation. J. Differ. Equ. 205, 365-389 (2004)

[26] Fila, M., Winkler, M., Yanagida, E.: Slow convergence to zero for a parabolic equation with a supercritical nonlinearity. Math. Ann. 340, 477-496 (2008)

[27] Fujita, H.: On the blowing up of solutions of the Cauchy problem for $u_{t}=$ $\Delta u+u^{1+\alpha}$. J. Fac. Sci. Univ. Tokyo Sect. I(13), 109-124 (1966)

[28] Galaktionov, V.A., Vázquez, J.L.: Continuation of blowup solutions of nonlinear heat equations in several space dimensions. Commun. Pure Appl. Math. 50, 1-67 (1997)

[29] Giga, Y., Miyakawa, T.: Navier-Stokes flow in $\mathbb{R}^{d}$ with measures as initial vorticity and Morrey spaces. Commun. Partial Differ. Equ. 14, 577-618 (1989)

[30] Granero-Belinchón, R., Orive-Illera, R.: An aggregation equation with a nonlocal flux. Nonlinear Anal., Theory Methods Appl., Ser. A 108, 260-274 (2014) 
[31] Gui, C., Ni, W.-M., Wang, X.: On the stability and instability of positive steady states of a semilinear heat equation in $\mathbb{R}^{n}$. Commun. Pure Appl. Math. 45, 1153-1181 (1992)

[32] Lemarié-Rieusset, P.-G.: Recent Developments in the Navier-Stokes Problem. Chapman \& Hall/CRC Research Notes in Mathematics 431, Boca Raton (2002)

[33] Lemarié-Rieusset, P.-G.: Small data in an optimal Banach space for the parabolic-parabolic and parabolic-elliptic Keller-Segel equations in the whole space. Adv. Differ. Equ. 18, 1189-1208 (2013)

[34] Lemarié-Rieusset, P.-G.: Sobolev multipliers, maximal functions and parabolic equations with a quadratic nonlinearity. J. Funct. Anal. 274, 659-694 (2018)

[35] Mizoguchi, N.: On the behavior of solutions for a semilinear parabolic equation with supercritical nonlinearity. Math. Z. 239, 215-229 (2002)

[36] Mizoguchi, N.: Boundedness of global solutions for a supercritical semilinear heat equation and its application. Indiana Univ. Math. J. 54, 1047-1059 (2005)

[37] Pilarczyk, D.: Asymptotic stability of singular solution to nonlinear heat equation. Disc. Cont. Dyn. Syst. 25, 991-1001 (2009)

[38] Pilarczyk, D.: Self-similar asymptotics of solutions to heat equation with inverse square potential. J. Evol. Equ. 13, 69-87 (2013)

[39] Poláčik, P., Yanagida, E.: On bounded and unbounded global solutions of a supercritical semilinear heat equation. Math. Ann. 327, 745-771 (2003)

[40] Quittner, P., Souplet, P.: Superlinear Parabolic Problems. Blow-Up, Global Existence and Steady States. Birkhäuser Advanced Texts, Basel (2007)

[41] Souplet, Ph: Morrey spaces and classification of global solutions for a supercritical semilinear heat equation in $\mathbb{R}^{n}$. J. Funct. Anal. 272, 2005-2037 (2017)

[42] Souplet, Ph, Weissler, F.B.: Regular self-similar solutions of the nonlinear heat equation with initial data above the singular steady state. Ann. Inst. Henri Poincaré, Anal. non Linéaire 20, 213-235 (2003)

[43] Sugitani, S.: On nonexistence of global solutions for some nonlinear integral equations. Osaka J. Math. 12, 45-51 (1975)

[44] Taylor, M.E.: Analysis on Morrey spaces and applications to Navier-Stokes and other evolution equations. Commun. Partial Differ. Equ. 17, 1407-1456 (1992)

Piotr Biler

Instytut Matematyczny

Uniwersytet Wrocławski

pl. Grunwaldzki $2 / 4$

50-384 Wrocław

Poland

e-mail: Piotr.Biler@math.uni.wroc.pl 
Dominika Pilarczyk

Wydział Matematyki

Politechnika Wrocławska

Wybrzeże Wyspiańskiego 27

50-370 Wrocław

Poland

e-mail: Dominika.Pilarczyk@pwr.edu.pl

Received: 11 July 2018.

Accepted: 22 January 2019. 